\title{
COMMENT
}

\section{DISCOUNTING HEALTH AND COST-EFFECTIVENESS ANALYSIS: A RESPONSE TO NORD}

\author{
JAMES K. HAMMITT ${ }^{\mathrm{a}, \mathrm{b}}$ \\ ${ }^{\mathrm{a}}$ Harvard University (Center for Risk Analysis), Boston MA, USA \\ ${ }^{\mathrm{b}}$ Toulouse School of Economics (LERNA-INRA), Toulouse, France
}

\begin{abstract}
SUMMARY
Nord (2011) criticizes standard arguments which assert that consistency requires that future health benefits must be discounted at the same rate as future costs in cost-effectiveness analysis (CEA). He suggests these arguments are misguided because they require transitivity of preferences across decision contexts and that it can be appropriate to discount health at different rates depending on the programs to be compared. I claim that rejecting transitivity is unwarranted and would sharply diminish the utility of CEA. Factors that tempt Nord to reject consistency can be accommodated by recognizing that CEA does not perfectly mimic normative social preferences because it omits factors (like distribution of health in a population) that can be normatively significant. A better approach is to maintain consistency in application of CEA but authorize decision makers to depart from rankings implied by CEA when justified and to explain which specific factors justify the decision. Finally, the assertion that health must be discounted at the same rate as costs requires the additional assumption that the dollar value of health does not change over time, a point that is not always recognized in standard arguments. Copyright (C) 2011 John Wiley \& Sons, Ltd.
\end{abstract}

Received 24 November 2010; Revised 8 April 2011; Accepted 15 June 2011

KEY WORDS: cost-effectiveness analysis; discount rate

\section{INTRODUCTION}

Erik Nord (2011) criticizes 'consistency arguments' that are often invoked to argue that future health benefits (typically measured in quality-adjusted life years (QALYs)) should be discounted at the same rate as future costs (measured in dollars or other monetary units). He writes that these arguments assume 'transitivity in preferences across decision contexts in which different considerations are invoked' and suggests that it may be appropriate to discount health at different rates depending on 'the comparator in question' (p. 19).

I believe this rejection of transitivity lies at the heart of the disagreement between Nord and the authors of the consistency arguments he criticizes. Many health economists believe that social preferences should be transitive across a larger set of decision contexts than Nord suggests and reject his claim that the rate at which health benefits are discounted should depend on the specific programs that are compared.

The stronger claim that consistency requires health to be discounted at the same rate as costs depends on an additional condition that is not always specified and is not normatively required: that the monetary value of health increments (the threshold value used to determine whether an intervention is cost-effective) does not change over time.

*Correspondence to: Center for Risk Analysis, Harvard University, 718 Huntington Ave., Boston, MA 02115 USA. E-mail: jkh@ harvard.edu 
The resolution of this disagreement has important implications for the use of cost-effectiveness analysis (CEA). In my view, CEA is useful to the extent that it helps rank programs in accord with normative social preferences (defined as the preferences society should have, even if they are not always respected). ${ }^{1}$ As I discuss later, CEA should not be treated as an inviolable decision rule because it does not incorporate all factors that are normatively significant. Incremental cost-effectiveness ratios (ICERs) are intended to quantify the efficiency with which resources can be converted into health through specific programs. If this measure of efficiency is not normatively significant, what is the purpose of computing it?

Cost-effectiveness analysis can mimic only transitive preferences. This follows because ICERs are real numbers and the equality and inequality relations (i.e., $>, \geq,=\leq,<$ ) applied to real numbers are transitive.

If normative social preferences are transitive across a wide range of decision contexts, then CEA can provide guidance for a correspondingly broad set of decisions. In this case, it can be extremely useful to conduct CEAs, to catalog them as in the CEA Registry (www.cearegistry.org) and to make them comparable across contexts (e.g., by measuring 'health' in QALYs rather than more natural units such as cases of particular diseases, by estimating QALY weights using a standard method such as the EQ-5D rather than study-specific methods, or by following the specification of the Washington panel's 'reference case,' Gold et al., 1996). Alternatively, if transitivity across a significant range of decision contexts is not normatively required, then CEA is likely to be much less useful and there is an urgent need to determine which decision contexts evoke sufficiently 'different considerations' that transitivity is not normatively required and hence CEA results may mislead decision makers.

Consider Nord's programs X, Y, Z, and W and the corresponding ICERs (compared with no program) displayed in the following table (in Nord's paper, $T=10)$. Each program incurs costs ( ' $\mathrm{C}$ ' or ' $\mathrm{C} /(1+\mathrm{r})^{\mathrm{T}}$ ) ) and provides health benefits $(\mathrm{H})$ in the indicated years. Let $\mathrm{s}$ and $\mathrm{d}$ be the rates at which costs and health are discounted, respectively, and let $r$ be the relevant interest rate, for example, the rate at which the government can shift resources between years 0 and $\mathrm{T}$ by increasing or retiring debt.

\begin{tabular}{lccl}
\hline Program & Year 0 & Year T & ICER \\
$X$ & $\mathrm{C}, \mathrm{H}$ & $\mathrm{C}$ & $\mathrm{C} / \mathrm{H}$ \\
$\mathrm{Y}$ & & $\mathrm{C}$ & $\mathrm{H}$ \\
$\mathrm{Z}$ & $\mathrm{C}$ & $\left.\mathrm{H}(1+\mathrm{s})^{\mathrm{T}}\right] /\left[\mathrm{H} /(1+\mathrm{d})^{\mathrm{T}}\right]$ \\
$\mathrm{W}$ & $\mathrm{C} /(1+\mathrm{r})^{\mathrm{T}}$ & $\mathrm{H}$ & $\mathrm{C} /\left[\mathrm{H} /(1+\mathrm{d})^{\mathrm{T}}\right]$ \\
\hline
\end{tabular}

Nord suggests that it is plausible for society to be simultaneously indifferent between the following pairs of policies:

$\mathrm{X}$ and $\mathrm{Y}$ (start-time neutrality)

$\mathrm{X}$ and $\mathrm{Z}$ (benefit-time neutrality)

$\mathrm{Y}$ and $\mathrm{W}$ (opportunity-cost of resources). ${ }^{2}$

The conditions under which the programs in each pair have equal ICERs are:

$\mathrm{d}=\mathrm{s}$ (health discounted at same rate as costs)

$\mathrm{d}=0$ (health discounted at rate 0 )

$\mathrm{s}=\mathrm{r}$ (costs discounted at interest rate).

These conditions cannot be satisfied simultaneously except in the unusual case that the interest rate $r$ is zero.

\footnotetext{
${ }^{1}$ If CEA is viewed as providing normative guidance, its methods are not invalidated by evidence that individuals or institutions make decisions that conflict with its findings (e.g., Kahneman and Tversky, 1979). If it does not provide normative guidance, what purpose does it serve?

${ }^{2}$ Indifference between $\mathrm{Y}$ and $\mathrm{W}$ arises from the assumption that $\mathrm{W}$ could be achieved by paying in advance for program $\mathrm{Y}$; that is, investing the amount $\left[C /(1+r)^{\mathrm{T}}\right]$ in year 0 to yield the amount $\mathrm{C}$ in year $\mathrm{T}$ then spending this amount on program $\mathrm{Y}$.
} 
Nord notes that the preferences specified in (1), (2), and (3) cannot be represented using CEA and a common discount rate for health. He suggests that the rate at which health benefits are discounted may depend on the decision context. Specifically, in comparing $\mathrm{X}$ with $\mathrm{Y}$, 'discounting from program start times to present time is not really necessary' but if discounting is applied, 'the rate will depend on start time differences.' In comparing $\mathrm{X}$ with $\mathrm{Z}$, 'the discount rate for benefits ... will depend on benefit time preferences and need not be consistent with the discount rate' used when comparing X with Y (p. 19). ${ }^{3}$

Nord argues that discounting health at different rates depending on whether $\mathrm{X}$ is compared with $\mathrm{Y}$ or with $\mathrm{Z}$ should not 'lead to inconsistency and confusion' for two reasons. First, 'prioritizing in health care normally has to do with distributing current resources,' which implies the choice between $\mathrm{X}$ and $\mathrm{Y}$ is rarely confronted. Second, when comparing $\mathrm{X}$ with Z, consideration of the potential beneficiaries' 'need comes into play' (p. 19). I address each reason in turn.

In response to the first point, although it may be true that the bureaucracies that manage government health systems must decide how to spend their exogenously determined budgets each year and cannot shift budgetary amounts between years, other decision makers do confront choices about allocating resources to health in different time periods and CEA can provide them with useful guidance. Legislators and treasury officials determine the annual budgets of government health-care systems and do shift budgetary costs through time (e.g., by borrowing or retiring debt). In addition, CEA has been advocated and is in some cases required for evaluating environmental, health, and safety regulations (e.g., US Office of Management and Budget, 2003). These regulations require firms and consumers to spend their resources to promote health. In this application, there is no fixed budget against which costs are assessed and the start time of the regulation is a government choice.

In response to the second point, CEA is a method that can provide useful guidance but it is unlikely to rank health programs perfectly in accord with normative social preferences. This follows because normative social preferences can depend on factors in addition to the consequences that are incorporated in CEA, such as the distribution of consequences among individuals. As a consequence, CEA should not be treated as an inviolable decision rule. Rather, decision makers should depart from the ranking implied by CEA when they judge it to be appropriate, but should justify the departure by explaining which additional factors are critical and why they are sufficiently important to justify the departure.

Nord suggests that a sufficient reason for indifference between programs $\mathrm{X}$ and $\mathrm{Z}$ (that provide benefits at different dates) is that potential beneficiaries 'are equally much in need of intervention now, even if the benefits occur at different times' (p. 19). He suggests that the implications of this factor can be accommodated by using different health-discount rates so that the resulting ICERs reflect normative social preferences. In my view, there are better approaches. First, if 'need of intervention' is a property that can be quantified, associated with specific times, and incorporated in either the numerator or denominator of an ICER, it should be. If not, it should be treated like other normatively significant factors that are not incorporated in CEA, that is, it should be a reason for departing from a CEA-based ranking in cases where it is judged sufficiently important.

Explicit acknowledgment of factors that influence normative social preferences but are not included in CEA is critical to evaluating programs. Indeed, failure to recognize the normatively significant difference between the beneficiaries of programs $\mathrm{Z}$ and $\mathrm{W}$ explains Nord's 'paradoxical result that $\mathrm{X} \ldots$ is equivalent to both $\mathrm{Z}$ and $\mathrm{W}$, even though $\mathrm{W}$ is clearly preferable to $\mathrm{Z}$ ' (p. 18). Programs $\mathrm{W}$ and $\mathrm{Y}$ are equivalent in the sense that they provide the same benefits to the same beneficiaries. But program $\mathrm{Z}$ is not equivalent to $\mathrm{W}$ and $\mathrm{Y}$ because its beneficiaries are different and are judged to be 'equally much in need of intervention now, even if the benefits occur at different times.' The symbol ' $\mathrm{H}$ ' in program $\mathrm{Z}$ does not represent the same benefits as the

\footnotetext{
${ }^{3}$ Nord introduces the concepts of start-time difference and benefit-time difference. Conventional CEA associates each consequence (cost or benefit) with the time it is realized. In his examples, start-time differences are equivalent to differences in when costs are incurred, but 'start time' suggests something different (if a program provides benefits sooner than costs, would start time and benefit time coincide?). When a decision (commitment) is made can be important in contexts where circumstances or information may change (there can be an option value to postponing decision). Without a more explicit definition of 'start time,' the implications of adopting the new terminology are not clear.
} 
symbol ' $\mathrm{H}$ ' in programs $\mathrm{W}$ and $\mathrm{Y}$ and hence it does not follow that ' $\mathrm{W}$ is clearly preferable to $\mathrm{Z}$.' Nor is it necessary to reject transitivity of preferences. The proposed indifference between $\mathrm{X}$ and $\mathrm{Z}$ is described more clearly by recognizing the normative significance of beneficiaries' need as a significant factor additional to the timing of benefits; the indifference between $\mathrm{X}$ and $\mathrm{Z}$ does not reflect pure 'benefit-time neutrality' (p. 18).

Although I argue that the discount rates used in CEA should not depend on which programs are being compared, that does not imply that health should be discounted at the same rate as costs. That stronger claim depends on the additional requirement that preferences exhibit start-time neutrality, or more generally that the rate of trade-off between costs and health within a period (the threshold ICER at which an intervention is judged 'cost-effective' or what Nord calls the 'dollar value of health,' p. 24) does not change over time (cf. Gravelle and Smith, 2001). A plausible alternative assumption is that the dollar value of health should increase over time (assuming society becomes richer in material goods). In that case, it would be appropriate to use larger threshold values in evaluating future programs and to discount health at a smaller rate than costs (including zero and possibly negative rates). ${ }^{4}$ When comparing current and future programs like $\mathrm{X}$ and $\mathrm{Y}$, there are three rates of concern (the discount rate for costs, the discount rate for health, and the rate at which the dollar value of health changes between year 0 and year $\mathrm{T}$ ) but only two degrees of freedom. Assuming that ICERs are to represent transitive preferences, these rates are mutually dependent: given the values of any two, the value of the third is determined. ${ }^{5}$ Consistency arguments that imply health should be discounted at the same rate as costs assume a constant dollar value of health without always acknowledging it.

In summary, CEA is a normative tool that can provide important guidance in choosing among health programs. It can be used in a broad set of decision contexts, including choices about coverage of preventative and treatment services by government health systems within and between periods and about regulations that require firms or individuals to spend resources to reduce health risks. By associating ICERs with programs and appropriate comparators and using consistent methods (that do not depend on the specific programs compared), CEA necessarily yields a transitive ranking. When factors not included in the ICER are sufficiently important that an alternative with a larger ICER is normatively socially preferred, that can be accommodated by making decision makers responsible to choose the socially preferred option and to explain which specific factors justify departure from the ranking provided by CEA. It is not necessary to limit use of CEA to "decision contexts in which [no] different considerations are invoked' (p. 19).

\section{ACKNOWLEDGEMENTS}

I thank Erik Nord for his stimulating presentation of this material at a Harvard seminar and for subsequent discussion. Financial support was provided by INRA and the European Research Council under the European Community's Seventh Framework Programme (FP7/2007-2013) Grant Agreement no. 230589.

Conflict of interest: There are no conflicts of interest. This work did not involve experiments with human subjects.

\footnotetext{
${ }^{4}$ Gravelle et al. (2007) and Claxton et al. (2011) analyze the case in which the dollar value of health increases over time but health expenditures are chosen using a threshold ICER that may not reflect these values.

${ }^{5}$ Using the notation in the table, the ratio of the dollar value of health in year $\mathrm{T}$ to the dollar value of health in year 0 is $[(1+\mathrm{d}) /(1+\mathrm{s})]^{\mathrm{T}}$. Gravelle et al. (2007) provide a similar result in their Equation (3).
} 
DISCOUNTING HEALTH AND COST-EFFECTIVENESS ANALYSIS

\section{REFERENCES}

Claxton K, Paulden M, Gravelle H, Brouwer W, Culyer AJ. 2011. Discounting and decision making in the economic evaluation of health-care technologies. Health Economics 20: 2-15.

Gold M, Siegel J, Russell LB, Weinstein WC (eds.), 1996. Cost-Effectiveness in Health and Medicine. Oxford University Press: Oxford.

Gravelle H, Brouwer W, Niessen L, Postma M, Rutten F. 2007. Discounting for economic evaluations: stepping forward toward optimal decision rules. Health Economics 16: 307-317.

Gravelle H, Smith DS. 2001. Discounting for health effects in cost-benefit and cost-effectiveness analysis. Health Economics 10: 587-599.

Kahneman D. Tversky A. 1979. Prospect theory: an analysis of decision under risk. Econometrica 47: $263-291$.

Nord E. 2011. Discounting future health benefits: the poverty of consistency arguments. Health Economics 20: 16-26.

US Office of Management and Budget, Circular A-4, Washington, D.C., September 17, 2003. 